\title{
Is risk of Kaposi's sarcoma in AIDS patients in Britain increased if sexual partners came from United States or Africa?
}

\author{
Valerie Beral, Diana Bull, H Jaffe, B Evans, N Gill, H Tillett, A J Swerdlow
}

\begin{abstract}
Objective-To determine whether the risk of Kaposi's sarcoma in patients with AIDS is increased by sexual contact with groups from abroad with a high incidence of Kaposi's sarcoma.

Design-Analysis of data on patients with AIDS and Kaposi's sarcoma.

Setting-United Kingdom.

Patients -2830 patients with AIDS reported to the Communicable Disease Surveillance Centre and the Communicable Disease (Scotland) Unit up to March 1990, of whom 566 had Kaposi's sarcoma.

Main outcome measures-Number of patients with Kaposi's sarcoma and possible source of HIV infection.
\end{abstract}

Results -537 of 2291 men (23\%) who acquired AIDS through sexual contact had Kaposi's sarcoma; the risk associated with heterosexual contact was $10 \%(14 / 135)$. None of the patients with Kaposi's sarcoma for whom information on source of infection was available had contracted AIDS through non-sexual means. Kaposi's sarcoma was more common among homosexual men whose likely source of infection included the United States (171/551, $31 \%)$ or Africa $(9 / 134,26 \%)$ than among those infected in the United Kingdom (119/625, 19\%; $\mathbf{p}<0.05$ ).

Conclusion-The data suggest that Kaposi's sarcoma is caused by a transmissible agent and that an important route of transmission is sexual contact.

Imperial Cancer Research Fund, Cancer

Epidemiology Unit, Gibson

Building, Radcliffe

Infirmary, Oxford

OX2 6HE

Valerie Beral, MRCP, director

Diana Bull, research officer

Institute of Cancer

Research, Chester Beatty

Laboratories, London

SW3 6JB

$\mathrm{H}$ Jaffe, MD, visiting scientist

Public Health Laboratory Service, AIDS Centre, Communicable Disease Surveillance Centre,

London NW9

B Evans, FFPHM, consultant epidemiologist

N Gill, FFPHM, consultant epidemiologist

H Tillett, PHD, statistician

London School of Hygiene and Tropical Medicine, London WC1E 7HT

A J Swerdlow, MD, senior lecturer

Correspondence to:

Dr Beral.

BMF 1991:302:624-5 infection. This information was combined to cre vossible source of mentioned and the United States and Africa were of special interest priority was given, in order, to the
United States, Africa, and other foreign countries. The country of possible source of HIV infection was classified as the British Isles only; the United States, with or without other countries; Africa, with or without other countries; or elsewhere.

The Communicable Disease Surveillance Centre's reporting form contains information on the clinical manifestations of AIDS, including whether Kaposi's sarcoma was diagnosed. Our data relate to Kaposi's sarcoma as an indicator disease that was present when AIDS was diagnosed, and the results are presented as percentages of patients with AIDS who had Kaposi's sarcoma. The effects of age, ethnic group, and year and place of diagnosis were modelled by using $\log$ linear regression methods. If a patient had multiple exposure categories for AIDS priority was given, in order, to homosexual contact, intravenous drug misuse, haemophilia, receipt of blood transfusions, and heterosexual contact.

\section{Results}

Of 2830 patients with AIDS reported to the surveillance centre up to March 1990, 566 had Kaposi's sarcoma reported as an indicator disease present when AIDS was diagnosed. The percentage with Kaposi's sarcoma varied considerably according to the patients' exposure patterns. One in four homosexual or bisexual men $(23 \%, 537 / 2291)$ were reported to have Kaposi's sarcoma; a similar risk was reported for homosexual injecting drug users $(24 \%, 9 / 38$ men $)$. One in 10 patients who acquired HIV by heterosexual contact were reported to have Kaposi's sarcoma $(10 \%, 14 / 135)$. The HIV transmission group was not stated for the remaining six patients with Kaposi's sarcoma. There were no cases of Kaposi's sarcoma among 83 injecting drug users, 163 people with haemophilia and clotting disorders, 47 transfusion recipients, and 23 children.

Among homosexual men the percentage with Kaposi's sarcoma declined regularly over time, from $38 \%(9 / 24)$ for those diagnosed with AIDS in 1983, to $34 \%(66 / 197)$ in $1985,23 \%(118 / 517)$ in 1987 , and $17 \%$ (77/454) in 1989. The percentage with Kaposi's sarcoma also varied with age with a peak at age $35-44$. The percentages were $17 \%(14 / 81), 24 \%(195 / 797), 26 \%$ (241/934), 21\% (77/372), and 14\% (15/111) respectively at ages 18-24, 25-34, 35-44, 45-54, and 55 and over. The percentage with Kaposi's sarcoma was twice as high in homosexual men whose condition was diagnosed in London $(26 \%, 466 / 1803)$ as it was in those diagnosed elsewhere in the United Kingdom $(15 \%, 79 / 523)$ and was similar in white patients $(23 \%, 474 / 2058)$ and black patients $(20 \%, 9 / 46)$.

Kaposi's sarcoma was also more common in homosexual men whose likely source of HIV infection included the United States or Africa rather than the British Isles $(p<0.05$, table). The higher percentage in those whose likely source included the United States rather than the British Isles was evident at all times (48\% (10/21) v 0\% (0/5) in $1982-3 ; 42 \%(56 / 133)$ v $31 \%$ $(18 / 59)$ in $1984-5 ; 27 \%(58 / 217) v 20 \%(49 / 246)$ in 1986-7; and $25 \%(37 / 148) v 17 \%(52 / 306)$ in $1988-9)$. The difference persisted and remained significant 
when we controlled simultaneously for age, ethnic group, and year and place of diagnosis of AIDS. For $771(33 \%)$ of the 2329 homosexual men the likely source of their HIV infection was not recorded, and $22 \%$ of these had Kaposi's sarcoma.

Frequency of Kaposi's sarcoma in patients who acquired HIV by sexual contact according to country reported as possible source of $H I V$ infection

\begin{tabular}{|c|c|c|}
\hline \multirow[b]{2}{*}{ Country } & \multicolumn{2}{|c|}{ No (\%) of patients with Kaposi's sarcoma } \\
\hline & $\begin{array}{l}\text { HIV transmitted by } \\
\text { homosexual contact }\end{array}$ & $\begin{array}{l}\text { HIV transmitted by } \\
\text { heterosexual contact }\end{array}$ \\
\hline $\begin{array}{l}\text { British Isles only } \\
\text { Abroad, including: }\end{array}$ & $119 / 625(19)$ & $0 / 20$ \\
\hline United States & $171 / 551(31)^{\star}$ & $3 / 8 \quad(38)$ \\
\hline Africa & $9 / 34 \quad(26)$ & $7 / 73(10)$ \\
\hline Elsewhere except United & & \\
\hline States or Africa & $77 / 348(22)$ & $0 / 12$ \\
\hline
\end{tabular}

*Includes 32 men ( 10 with Kaposi's sarcoma) for whom both the United States and Africa were reported as possible sources of HIV infection.

Of the patients who acquired HIV by heterosexual contact, $99 \%$ had the country of origin of their likely source of HIV infection recorded. Among the 114 whose ethnic group was recorded Kaposi's sarcoma was largely confined to patients reporting Africa as the possible source of their HIV infection (table). All seven cases of Kaposi's sarcoma among those whose sexual partners were from Africa were black patients (7/54), and among them the percentage with Kaposi's sarcoma was similar for men $(13 \%, 4 / 32)$ and women $(14 \%$, $3 / 22$ ). There were three cases of Kaposi's sarcoma reported among eight patients who reported the United States as the possible source of their HIV infection. All three were white men.

\section{Discussion}

The characteristics of patients with AIDS and Kaposi's sarcoma in the United Kingdom and the United States are similar.' In both countries Kaposi's sarcoma was more common among those who acquired HIV sexually rather than parenterally and was most common in homosexual or bisexual men. Among homosexual or bisexual men with AIDS the age distribution and the decline in the percentage with Kaposi's sarcoma over time were similar in both countries.' In the United Kingdom Kaposi's sarcoma was reported most often in men from London and in the United States in men from California and New York, the focus of the AIDS epidemic.

The new findings in the United Kingdom relate to the country of origin of the patients' sexual partners. Kaposi's sarcoma was more common in the homosexual men whose reported source of HIV infection and thus whose sexual partners included men from the United States or Africa rather than the British Isles alone. In those who acquired HIV by heterosexual contact Kaposi's sarcoma was rare except among patients reported to have sexual contacts from Africa.

These results are based on surveillance data. Errors or omissions in reporting would obscure true differences rather than create spurious associations unless reporting was selective or biased. The only group in which important reporting biases may have occurred is the three white men who reported that they acquired HIV by heterosexual spread from the United States. These men may have been misclassified as United States data suggest that white men in the United States who report that they acquired HIV by heterosexual contact may have acquired it by homosexual activity.' Using Kaposi's sarcoma as an indicator disease for AIDS should not bias the findings as it is the presenting symptom in $77 \%$ of patients when it occurs. ${ }^{4}$

Overall, these findings support the hypothesis that Kaposi's sarcoma is caused by a transmissible agent and that an important mode of transmission is sexual. Furthermore, they suggest that the agent was introduced into the British homosexual population mainly from the United States and that it is not prevalent among British heterosexuals except those with sexual contacts from Africa.

We thank Sarah Jones for typing the manuscript. Harold Jaffe was on sabbatical leave from the Centers of Disease Control, Atlanta, Georgia

1 Beral V, Peterman TA, Berkelman RL, Jaffe HW. Kaposi's sarcoma among persons with AIDS: a sexually transmitted infection? Lancet 1990;335:123-8. Friedman-Kien AE, Saltzman BR, Cao Y, et al. Kaposi's sarcoma in HIV negative homosexual men. Lancet 1990;335:168-9.

3 Public Health Laboratory Service AIDS Centre. Human immunodeficiency virus infection in the United Kingdom: quarterly report 3 . The epidemic to virus infection in the United Kingdom: quarterly report 3 . The epidemic to
31 March 1988, with special reference to persons injecting drugs. $\mathcal{F}$ Infect 31 March 1988, with special reference to persons injecting drugs. $\mathcal{F}$ Infect
1989:18:77-88.

4 Lifson AR, Darrow WW, Hassol NA, et al. Kaposi's sarcoma in a cohort of homosexual and bisexual men. Am F Epidemiol 1990;131:221-31.

(Accepted 12 December 1990)
Monash University

Department of Obstetrics and Gynaecology, Monash Medical Centre, Clayton, Australia 3168

T Y Khong, MD, visiting research fellow

D L Healy, FRACOG, professor

\section{Department of}

Mathematics, Monash

University, Clayton,

Australia 3168

P I McCloud, PHD, lecturer

Correspondence to: $\operatorname{Dr} T$ Y Khong, Department of

Paediatric Pathology, John

Radcliffe Hospital, Oxford OX3 9DU.

BMF 1991;302:625-6

\section{Pregnancies complicated by abnormally adherent placenta and sex ratio at birth}

\section{T Y Khong, D L Healy, P I McCloud}

The ratio of male to female infants at birth is increased in women with pre-eclampsia or who have a spontaneous abortion.' Although the morphology of placentation in these conditions is diametrically different from that in placenta accreta, ${ }^{2}$ the sex ratio has not been examined in women with placenta accreta. We determined the sex ratio of infants born to women requiring manual removal of the placenta as this indicates a degree of accretion.

\section{Subjects, methods, and results}

We ascertained the sex of all singleton infants born at one hospital over 30 years (1959-88) by reviewing the delivery suite's birth register. The information was stratified according to gravidity for the first 10 years. The data were analysed by logistic regression. The log likelihood ratio was used to test the goodness of fit of the proposed models and to test hypotheses. This statistic is asymptotically $\chi^{2}$.

The sex ratio for all singleton infants was $1 \cdot 071$. A linear logistic model showed no significant difference among the proportions of girls born to women of gravidity $1,2,3$, or $\geqslant 4\left(\mathrm{p}>0.5 ; \chi^{2}=2 \cdot 67, \mathrm{df}=3\right)$ (table). The incidence of manual removal of the placenta was $3 \cdot 1 \%(3195 / 103462)$. The proportion of women requiring manual removal of the placenta was equal in all the gravidity groups $\left(p>0.5 ; \chi^{2}=0.85\right.$, $\mathrm{df}=3)$, with no trend with increasing gravidity $(\mathrm{p}>0.5$; $\chi^{2}=0 \cdot 4, \mathrm{df}=1$ ).

Among the women who required manual removal of the placenta the overall sex ratio was 0.844 , which was significantly less than that $(1.079)$ in the reference population $\left(\mathrm{p}<0.001 ; \chi^{2}=12 \cdot 49, \mathrm{df}=1\right)$. The proportions of boys born to the women who did and did not require manual removal of the placenta were 0.458 ( $95 \%$ confidence interval 0.440 to 0.475 ) and 0.519 